\section{AB0188 ACTIVATED PLATELETS ARE INCREASED IN CIRCULATION OF PATIENTS WITH SYSTEMIC SCLEROSIS AND ASSOCIATED WITH CLINICAL CHARACTERISTICS}

H. Yasuoka ${ }^{1}$, K. Sakata ${ }^{1}$, K. Yoshimoto ${ }^{1}$, K. Yamaoka ${ }^{1}$, M. Kuwana ${ }^{2}$, T. Takeuchi ${ }^{1}$ ${ }^{1}$ Keio University School of Medicine; ${ }^{2}$ Nippon Medical School Graduate School of Medicine, Tokyo, Japan

Background: Systemic sclerosis (SSc) is a systemic connective tissue disease characterised by excessive fibrosis, microvascular injury and autoantibody production 1), but mechanisms of disease process are still under investigation Recent studies focused on the role of circulating blood cells in the pathogenesis, especially lymphocytes 2,3 ) or monocytes 4,5 ), however other cellular components are not well-examined. Platelets play a significant role in hemostasis physiologically. However, recent studies have revealed that platelets contain various kinds of humoral factors such as cytokines, chemokines and growth factors and can distribute systemically through circulation and contribute to the disease process through activation and release of these factors 6).

Objectives: To elucidate the role of platelets in the pathogenesis of SSc, activation status of circulating platelets in patients with SSc and association with clinical characteristics were examined.

Methods: Twenty-one patients with SSc who fulfilled 2013 ACR/EULAR classification criteria and 16 healthy controls were involved. Platelets or microparticles (MPs) were defined as vesicles in platelet-rich plasma which is more or less than $1 \mu \mathrm{m}$ in diameter by forward and side scatter, respectively, and positive staining with anti-CD41 antibody using flow cytometry. Activation status of platelets was examined by the expression of activation markers on platelets such as P-selectin (CD62P) or activated glycoprotein Ilb/llla (PAC1). Production of microparticles (MPs) is defined as ratio of proportion of MP to that of platelets. Release reaction of platelets was evaluated by release of platelet factor 4 (PF4) in culture supernatant of coculture with skin fibroblasts using enzyme-linked immunosorbent assay (ELISA). Association or correlation between proportion of activated platelets and clinical characteristics or parameters of patients with scleroderma was also examined.

Results: As for the characteristics of 21 patients with SSc, male to female ratio was 3: 18 , proportion of diffuse cutaneous SSc was $24 \%$, mean age was $63 \pm 13$ years, and mean disease duration was $16 \pm 13$ years. In SSc, both proportion of CD62p+or PAC1 +activated platelets $(p<0.05, p<0.05$, respectively) and production of MP were higher $(p<0.05)$ compared to those in healthy controls. Of these, proportion of CD62p+platelets and MP production were correlated each othe $(r=0.88, p<0.05)$. Also, release reaction of platelet was upregulated in platelets from patient with SSc compared to healthy controls $(p<0.05)$. When activation status of platelets was compared to clinical parameters, both proportion of CD62p +activated platelets and MP production were higher in diffuse cutaneous SSC $(p<0.05, p<0.05$, respectively) and correlated with modified Rodnan skin score $(p<0.05, p<0.05$, respectively).

Conclusions: In SSc, proportion of activated platelets were higher and associated with skin sclerosis, suggesting the involvement in the pathogenesis of SSc.

\section{REFERENCES :}

[1] Denton CP, et al. Nat Rev Rheumatol. 2013;9(8):451-64.

[2] Almeida I, et al. 2015;49(3):347-69.

[3] Yoshizaki A. Immunol Lett. 2018.

[4] Moleno-Moral A, et al. Ann Rheum Dis. 2018.

[5] Masuda A, et al. Arthritis Res Ther. 2013;15:R74.

[6] Semple JW, et al. Nat Rev Immunol. 2011;11(4):264-74.

Disclosure of Interest: None declared

DOI: 10.1136/annrheumdis-2018-eular.6162

\section{AB0189 3D SKIN ORGANOID MIMICKING SYSTEMIC SCLEROSIS GENERATED BY PATIENT-DERIVED INDUCED PLURIPOTENT STEM CELLS: 'DISEASE IN A DISH' AND} DEVELOPMENT OF ANIMAL MODEL

J.-W. Kim ${ }^{1}$, Y. Kim ${ }^{2}$, J. Kim², M.-J. Park ${ }^{3}$, E. Kwon ${ }^{1}$, J. Lee ${ }^{1}$, S.-K. Kwok ${ }^{1}$, J. H. Ju', ${ }^{1,2}$ S.-H. Park ${ }^{1}{ }^{1}$ Division of Rheumatology, Department of Internal Medicine, School of Medicine, The Catholic University of Korea, Seoul St. Mary's Hospital; ${ }^{2}$ Catholic iPSC Research Center, College of Medicine, The Catholic University of Korea; ${ }^{3}$ Rheumatism Research Center, Catholic Research Institute of Medical Science, The Catholic University of Korea, Seoul, Korea, Republic of Ireland

Background: Systemic sclerosis (SSc) is a rare autoimmune disease characterised by vasculopathy and fibrosis of various organs including skin. Although SSc has high morbidity and mortality, evidences for disease modifying treatment are still lacking due to difficulties in performing clinical trials. Patient-specific induced pluripotent stem cells (iPSCs), which can differentiate into various cell types, are used in $3 \mathrm{D}$ organoid formation.

Objectives: We generated 3D skin organoid model from SSc-derived PSCs by differentiating them into keratinocytes and fibroblasts. SSc-mimicking 3D skin organoid can be used in studies for disease modelling and drug screening.

Methods: Peripheral blood mononuclear cells (PBMCs) from patients with SSc were reprogrammed to iPSCs. SSc-derived iPSCs differentiated into keratinocytes and fibroblasts in vitro. Expression of markers for iPSCs, keratinocytes, and fibroblasts were determined by reverse transcription polymerase chain reaction (RT-PCR) analysis and immunofluorescence assay (IFA). 3D skin organoid using iPSC-derived differentiation cell line was generated by 3D culture system. Histologic analysis was performed on 3D skin organoid. SSc-derived 3D skin organoid was applied to SCID skin defect mice. Histologic analysis was also performed on SCID skin graft model.

Results: SSc-derived iPSCs formed colonies that resemble embryonic stem cells. Alkaline phosphatase staining showed undifferentiated state of iPSCs Expression of iPSC markers was increased on SSc-iPSCs. Differentiated keratinocytes and fibroblasts from iPSCs highly expressed their markers for keratinocytes and fibroblasts, respectively. Dermis of SSc-derived 3D skin organoid was thicker and denser than that derived from healthy control. Epidermis and dermis of SCID skin graft model were thickened in those derived from SSc compared to those derived from healthy control.

Conclusions: Patient-derived 3D skin organoid and animal model well represented the characteristics of SSc. These models can serve as useful research tools to understand the disease and screen new drugs for SSc.

Disclosure of Interest: None declared

DOI: 10.1136/annrheumdis-2018-eular.4502

\section{AB0190 IMPAIRED ACTIVATION OF ATAXIA-TELANGIECTASIA MUTATED PROTEIN KINASE IN IMMUNE CELLS IS ASSOCIATED WITH CLINICAL FEATURES IN PATIENTS WITH SYSTEMIC SCLEROSIS}

K. Sakata ${ }^{1}$, H. Yasuoka ${ }^{1}$, K. Yoshimoto $^{1,2}$, K. Yamaoka $^{1}$, T. Takeuchi $^{1}$ ${ }^{1}$ Department of Internal Medicine, Division of Rheumatology, Keio University School of Medicine; ${ }^{2}$ Clinical and Translational Research Center, Keio University Hospital, Tokyo, Japan

Background: Ataxia-telangiectasia mutated (ATM) is a protein kinase associated with ataxia-telangiectasia (AT), which is an autosomal recessive disorder due to defective functional activity of ATM. Telangiectasia, seen in AT, is also well known as one of the major characteristics of systemic sclerosis (SSc). ATM plays an important role not only in DNA damage repairing system, but also in the process of regulation of oxidative stress. ${ }^{1}$ Moreover, it has been reported that oxidative stress may contribute to disease process of $\mathrm{SSc}^{23}$. Based on these background, we hypothesised that ATM may play a substantial role in the pathogenesis of SSc. However, the possible association between ATM activity and SSc development is not fully understood.

Objectives: To clarify the role of ATM in the pathogenesis of SSc, we demonstrated the expression and activation level of ATM in circulating immune cells and analysed the association with clinical characteristics of the patients.

Methods: Whole blood samples were collected from twenty-four patients with SSc and 12 healthy controls (HC). Expression levels of total ATM and active phosphorylated ATM (pATM) were examined in each immune cell subset (neutrophil, monocyte, T cell, B cell and NK cell) by mean fluorescence intensity (MFI) using flow cytometer. Each MFI level of ATM and PATM was compared between patients with SSc and $\mathrm{HC}$, and was analysed the correlation with clinical characteristics of SSc patients, retrospectively collected from patients' records.

Results: The expression level of pATM was significantly lower in monocytes, neutrophils, and T cells in SSc as compared with ${ }^{\mathrm{HC}}(1887 \pm 128$ vs $2386 \pm 181$, $p=0.03 ; 10265 \pm 861$ vs $16087 \pm 1218, p=0.0004 ; 1326 \pm 73$ vs $1675 \pm 103, p=0.009$; respectively), whereas no significant difference in total ATM level was observed in each cell subset between two groups. Notably, the expression levels of pATM in monocytes of the patients with interstitial lung disease (ILD) was lower than that of the patients without ${ }^{L D}(1663 \pm 136$ vs $1999 \pm 96, p=0.05)$. Furthermore, there was a tendency of correlation between PATM level in monocyte and parameters of pulmonary function test, such as forced vital capacity. No significant differences and/ or correlation between PATM expression level in other cell subsets and clinical parameters of the patients, such as SSc subtype, SSc-specific autoantibodies, presence of pulmonary arterial hypertension, gastrointestinal involvement, digital tip ulcer, pitting scar and modified Rodnan skin score were observed.

Conclusions: In SSc, phosphorylated level of ATM in monocytes, neutrophils, and T cells was significantly lower than that of HC. Importantly, we found that ATM activation was impaired in monocyte of the SSc patients with ILD. These results 